\title{
Dynamical gap from holography in the charged dilaton black hole
}

\author{
Xiao-Mei Kuang ${ }^{1}$, Bin Wang ${ }^{1}$, and Jian-Pin $\mathrm{Wu}^{2,3,4}$ \\ ${ }^{1}$ INPAC, Department of Physics and Shanghai Key Lab for Particle Physics and Cosmology, \\ Shanghai Jiao Tong University, Shanghai 200240, China \\ ${ }^{2}$ Department of Physics, Hanyang University, Seoul 133-791, Korea \\ ${ }^{3}$ Center for Quantum Spacetime, Sogang University, Seoul 121-742, Korea \\ ${ }^{4}$ Department of Physics, School of Mathematics and Physics, Bohai University, JinZhou, 121013, China
}

\begin{abstract}
We study the holographic non-relativistic fermions in the presence of bulk dipole coupling in charged dilatonic black hole background. We explore the nontrivial effects of the bulk dipole coupling, the fermion charge as well as the dilaton field on the flat band, the Fermi surface and the emergence of the gap by investigating the spectral function of the non-relativistic fermion system. In particular, we find that the presence of the flat band in the non-relativistic case will suppress the Fermi momentum. Besides, we observe that the effect of the dipole coupling in the dilaton gravity is more explicit. Finally, we consider the non-relativistic fermions at nonzero temperature. A phase transition from insulator to a conducting state is observed as the fermion system becomes hotter.
\end{abstract}

PACS numbers: 11.25.Tq, 04.50.Gh, 71.10.-w 


\section{INTRODUCTION}

The AdS/CFT correspondence is a powerful method to investigate the strongly coupled many body phenomena by relating certain interacting quantum field theories to classical gravity systems. An interesting application of such duality is the study of condensed matter physics from the remarkable connection in gravitational physics, for reviews see for examples [1] 3$]$.

Stimulated by the AdS/CFT correspondence, the simplest realization to construct gravitational duals of the transition from normal state to superconducting state is to deal with Einstein's gravitational theory with a negative cosmological constant coupled to a gauge field. This idea caught some interesting properties of the realistic superconductor by discussing holography in the simplest charged AdS black hole background. However, the RN-AdS black hole contains nonzero entropy even at zero temperature. This situation was improved by changing the background bulk geometry to include a real scalar field, the dilaton, which allows zero entropy at zero temperature [4 6]. At zero temperature, it was argued that states of matter can be holographically described by a spacetime with an asymptotically $A d S_{4}$ Einstein-Maxwell-dilaton theory, the quantum phase transitions between different phases were exhibited [7]. More holographic properties disclosed in the dilaton black holes can be found in [5].

In addition to modifying the bulk gravitational backgrounds, recently there have been some attempts to modify the boundary conditions in describing the condensed matter system. In [8], it was shown that one can implement the holographic non-relativistic fermionic fixed points by imposing Lorentz breaking boundary conditions instead of the Lorentz covariant one on the Dirac spinor field, which can lead to the presence of an infinite flat band in the boundary field theory. Further studies on the holographic non-relativistic fermionic fixed points have been reported in [9, 10]. In [11, 12], the holographic non-relativistic fermionic fixed points were studied in the charged dilatonic black hole and black brane.

To capture more general features and phenomena relating to condensed matter physics, there have been a lot of investigations to consider a quantum field theory which contains fermions charged under a global U(1)

symmetry 13 20]. However, many of these studies concentrated on the fermions minimally coupled to gravity and gauge fields. Recently, introducing the coupling between the fermion and gauge field through a dipole interaction in the bulk charged AdS black hole background, it was remarkably found that as the strength of the interaction is varied, spectral density is transferred and beyond the critical interaction strength a gap 
opens up[21]. The existence of Fermi surfaces as the varying of the dipole coupling was also disclosed[22]. The extensions of the investigation on the dipole coupling to different bulk backgrounds were reported in [23 25].

In this work we will extend the study of the dipole interaction to the charged dilatonic AdS black hole background. Considering that different from the charged AdS black hole, the charged dilatonic AdS black hole has vanishing entropy in the low temperature limit, it is a suitable bulk background to study the holographic Fermi liquid. Furthermore, the dilaton can couple directly not only to the gauge field but also to the charged scalar, which can help to further disclose the role played by the dipole coupling in the boundary theory of Fermi liquid. In our study, instead of keeping Lorentz invariance for the boundary theory, we will impose Lorentz violating boundary terms for a spinor field following [8]. We will investigate the holographic spectral function behaviors at the non-relativistic fermionic fixed points and compare with the situations at the relativistic fermonic fixed point [24]. We will disclose how the effects of dilaton, the dipole coupling as well as the fermion charge modify the properties of Fermi gap, Fermi momentum etc. in the Fermi system.

The organization of this paper is as follows. In section II, we will derive the bulk Dirac equations in 4dimensional charged dilatonic AdS black hole. Then we will briefly discuss the holographic calculations of the retarded Green functions of those fermionic operators for non-relativistic theory. In section III, we will present our numerical results for the non-relativistic fixed point in different cases. Finally, we will summarize our results in the last section.

\section{HOLOGRAPHIC SETUP}

In this section, we will derive the Dirac equations of the bulk fermion coupling to the gauge field through dipole interaction in the charged dilatonic AdS black hole background. We will employ the Lorentz violating boundary term for the spinor field and study the holographic fermionic systems through the perturbations on the non-relativistic fermionic fixed point.

\section{A. Dirac equation}

We consider the non-minimal coupling between the spin-1/2 fermions and the gauge field in the form of the dipole interaction described by the bulk action

$$
S_{b u l k}=i \int d^{d} x \sqrt{-g} \bar{\zeta}\left(\Gamma^{a} \mathcal{D}_{a}-m-i p \not{F}\right) \zeta
$$


where $m$ and $p$ are the mass of the fermion field and the dipole coupling parameter, respectively. In the action, $\Gamma^{a}=\left(e_{\mu}\right)^{a} \Gamma^{\mu}, \not{f}=\frac{1}{4} \Gamma^{\mu \nu}\left(e_{\mu}\right)^{a}\left(e_{\nu}\right)^{b} F_{a b}$ and $\mathcal{D}_{a}=\partial_{a}+\frac{1}{4}\left(\omega_{\mu \nu}\right)_{a} \Gamma^{\mu \nu}-i q A_{a}$, with $\Gamma^{\mu \nu}=\frac{1}{2}\left[\Gamma^{\mu}, \Gamma^{\nu}\right]$ and the spin connection $\left(\omega_{\mu \nu}\right)_{a}=\left(e_{\mu}\right)^{b} \nabla_{a}\left(e_{\nu}\right)_{b}$, where $\left(e_{\mu}\right)^{a}$ form a set of orthogonal normal vector bases [26].

We intend to work in the charged dilatonic AdS black hole background with the metric

$$
\begin{array}{r}
d s^{2}=-g_{t t} d t^{2}+g_{x x}(d \vec{x})^{2}+g_{r r} d r^{2}=e^{2 B}\left[-f d t^{2}+(d \vec{x})^{2}\right]+\frac{1}{e^{2 B}} \frac{d r^{2}}{f}, \quad F=d A, \quad \phi=\frac{1}{2} \ln \left(1+\frac{Q}{r}\right) \\
B=\ln \frac{r}{L}+\frac{3}{4} \ln \left(1+\frac{Q}{r}\right), \quad f=1-\frac{\nu L^{2}}{(Q+r)^{3}}, \quad A_{a}=\left(\frac{\sqrt{3 Q \nu}}{Q+r}-\frac{\sqrt{3 Q} \nu^{\frac{1}{6}}}{L^{\frac{2}{3}}}\right)(d t)_{a}
\end{array}
$$

which is a solution to the Einstein-Maxwell-Dilaton action in 4-dimensional spacetime [4]

$$
S_{g}=\frac{1}{16 \pi G} \int_{M} d^{4} x \sqrt{-g}\left[R-\frac{1}{4} e^{\phi} F_{a b} F^{a b}-\frac{3}{2} \nabla_{a} \phi \nabla^{a} \phi+\frac{6}{L^{2}} \cosh \phi\right] .
$$

Here, $R$ and $L$ are the Ricci scalar and the AdS radius, respectively. $\phi$ denotes the dilaton field and $F=d A$ is the field strength of $U(1)$ gauge field. The temperature of the charged dilaton black hole and chemical potential near the boundary read

$$
T=\frac{\left.g_{t t}^{\prime}\right|_{r=r_{+}}}{4 \pi}=\frac{3 \nu^{\frac{1}{6}}}{4 \pi L^{\frac{5}{3}}} \sqrt{r_{+}}, \quad \mu=-\frac{\sqrt{3 Q} \nu^{\frac{1}{6}}}{L^{\frac{2}{3}}}
$$

where $r_{+}$is the black hole horizon with the form $r_{+}=\nu^{\frac{1}{3}} L^{\frac{2}{3}}-Q$ obtained from $f\left(r_{+}\right)=0$. When $\nu=\frac{Q^{3}}{L^{2}}$, the black hole has zero temperature.

The Dirac equation $(\mathcal{D}-m-i p \not{F}) \zeta=0$ in the bulk has the form

$$
\left(\sqrt{g^{r r}} \Gamma^{r} \partial_{r}-m-\frac{i p}{2} \sqrt{g^{r r} g^{t t}} \Gamma^{r t} \partial_{r} A_{t}\right) F-i\left(\omega+q A_{t}\right) \sqrt{g^{t t}} \Gamma^{t} F+i k \sqrt{g^{x x}} \Gamma^{x} F=0
$$

after taking the ansatz $\zeta=\left(-g g^{r r}\right)^{-\frac{1}{4}} F e^{-i \omega t+i k_{i} x^{i}}$ and setting $k_{i}=k \delta_{1}^{i}$ without loss of generality. It is convenient to express $F$ into $F=\left(F_{1}, F_{2}\right)^{T}$ and choose the following basis for our gamma matrices [29]

$$
\Gamma^{r}=\left(\begin{array}{cc}
-\sigma^{3} & 0 \\
0 & -\sigma^{3}
\end{array}\right), \quad \Gamma^{t}=\left(\begin{array}{cc}
i \sigma^{1} & 0 \\
0 & i \sigma^{1}
\end{array}\right), \quad \Gamma^{1}=\left(\begin{array}{cc}
-\sigma^{2} & 0 \\
0 & \sigma^{2}
\end{array}\right), \quad \Gamma^{2}=\left(\begin{array}{cc}
0 & -i \sigma^{2} \\
i \sigma^{2} & 0
\end{array}\right)
$$

The Dirac equation can be rewritten into

$$
\begin{array}{r}
\sqrt{g^{r r}} \partial_{r}\left(\begin{array}{c}
F_{1} \\
F_{2}
\end{array}\right)+m \sigma^{3} \otimes\left(\begin{array}{l}
F_{1} \\
F_{2}
\end{array}\right)=\sqrt{g^{t t}}\left(\omega+q A_{t}\right) i \sigma^{2} \otimes\left(\begin{array}{l}
F_{1} \\
F_{2}
\end{array}\right) \mp k \sqrt{g^{x x}} \sigma^{1} \otimes\left(\begin{array}{l}
F_{1} \\
F_{2}
\end{array}\right) \\
-p \sqrt{g^{t t} g^{r r}} \partial_{r} A_{t} \sigma^{1} \otimes\left(\begin{array}{c}
F_{1} \\
F_{2}
\end{array}\right) .
\end{array}
$$

Furthermore, to decouple the equation of motion, we take the decomposition $F_{ \pm}=\frac{1}{2}\left(1 \pm \Gamma^{r}\right) F$ with

$$
F_{+}=\left(\begin{array}{c}
0 \\
\mathcal{B}_{1} \\
0 \\
\mathcal{B}_{2}
\end{array}\right), \quad F_{-}=\left(\begin{array}{c}
\mathcal{A}_{1} \\
0 \\
\mathcal{A}_{2} \\
0
\end{array}\right), \quad \text { and } \quad F_{\alpha} \equiv\left(\begin{array}{c}
\mathcal{A}_{\alpha} \\
\mathcal{B}_{\alpha}
\end{array}\right), \alpha=1,2
$$


Under such decomposition, the Dirac equation (7) can be divided into

$$
\begin{aligned}
& \left(\sqrt{g^{r r}} \partial_{r} \pm m\right)\left(\begin{array}{c}
\mathcal{A}_{1} \\
\mathcal{B}_{1}
\end{array}\right)= \pm\left(\omega+q A_{t}\right) \sqrt{g^{t t}}\left(\begin{array}{c}
\mathcal{B}_{1} \\
\mathcal{A}_{1}
\end{array}\right)-\left(k \sqrt{g^{x x}}+p \sqrt{g^{t t} g^{r r}} \partial_{r} A_{t}\right)\left(\begin{array}{c}
\mathcal{B}_{1} \\
\mathcal{A}_{1}
\end{array}\right) \\
& \left(\sqrt{g^{r r}} \partial_{r} \pm m\right)\left(\begin{array}{c}
\mathcal{A}_{2} \\
\mathcal{B}_{2}
\end{array}\right)= \pm\left(\omega+q A_{t}\right) \sqrt{g^{t t}}\left(\begin{array}{c}
\mathcal{B}_{2} \\
\mathcal{A}_{2}
\end{array}\right)+\left(k \sqrt{g^{x x}}-p \sqrt{g^{t t} g^{r r}} \partial_{r} A_{t}\right)\left(\begin{array}{c}
\mathcal{B}_{2} \\
\mathcal{A}_{2}
\end{array}\right)
\end{aligned}
$$

It is straightforward to reduce the above two equations into the flow equation of $\xi_{I} \equiv \frac{\mathcal{A}_{I}}{\mathcal{B}_{I}}(I=1,2)$

$$
\left(\sqrt{f} e^{B} \partial_{r}+2 m\right) \xi_{I}=\left[v_{-}+(-1)^{I} e^{-B} k\right]+\left[v_{+}-(-1)^{I} k e^{-B}\right] \xi_{I}^{2}
$$

where $v_{ \pm}=\frac{e^{-B}}{\sqrt{f}}\left[\omega+q A_{t}\right] \pm p A_{t}^{\prime}$.

Near the AdS boundary, from (77) we see that the reduced Dirac field behaves as

$$
F_{I} \stackrel{r \rightarrow \infty}{\approx} a_{I} r^{-m L}\left(\begin{array}{l}
1 \\
0
\end{array}\right)+b_{I} r^{m L}\left(\begin{array}{l}
0 \\
1
\end{array}\right), \quad I=1,2
$$

Then the value $G_{I}=\frac{a_{I}}{b_{I}}$ can be expressed in the form

$$
G_{I}=\lim _{r \rightarrow \infty} r^{2 m} \xi_{I}
$$

Thus, we can read off $G_{I}$ by solving the flow equation (11) with the boundary conditions at the horizon[11]

$$
\xi_{I}= \begin{cases}i & \text { for } \omega \neq 0 \\ (-1)^{I} \operatorname{sign}(k) & \text { for } \omega=0\end{cases}
$$

It is worth indicating that from the flow equation (11) and the above boundary conditions, we can find $G_{I}$ with the following symmetries:

$$
\begin{aligned}
G_{1}(\omega, k) & =G_{2}(\omega,-k) ; \\
G_{1}(\omega, k ; q, p) & =-G_{2}^{*}(-\omega, k ;-q,-p) .
\end{aligned}
$$

Especially, when $m=0, G_{1}$ and $G_{2}$ satisfy the relation

$$
G_{1}(\omega, k)=-\frac{1}{G_{2}(\omega, k)}
$$

\section{B. Non-relativistic fermionic fixed point}

Most available works on the holographic fermionic systems focus on the perturbations on the relativistic fixed point by keeping the Lorentz invariance for the boundary theory. In [8], the authors first considered the boundary term by dropping the Lorentz invariance while still keeping the $U(1)$ global symmetry $\psi \rightarrow e^{i \theta} \psi$, 
the rotational invariance as well as the scale invariance. In this spirit, the boundary term to the bulk action (1) reads

$$
S_{b d y}=\frac{1}{2} \int_{\partial \mathcal{M}} d^{3} x \sqrt{-g g^{r r}} \bar{\zeta} \Gamma^{1} \Gamma^{2} \zeta
$$

This boundary term keeps the variational principle well-defined.

The variation of the on-shell action for the Dirac spinor has the form

$$
\delta S_{D}=\delta S_{b u l k}+\delta S_{b d y}=-\int d^{3} x\left(\delta B_{+}^{\dagger} A_{+}+B_{-}^{\dagger} \delta A_{-}+A_{+}^{\dagger} \delta B_{+}+\delta A_{-}^{\dagger} B_{-}\right)
$$

with $\left(A_{+}, A_{-}\right)=\frac{1}{\sqrt{2}}\left(\mathcal{A}_{1}+\mathcal{A}_{2}, \mathcal{A}_{1}-\mathcal{A}_{2}\right)$ and $\left(B_{+}, B_{-}\right)=\frac{1}{\sqrt{2}}\left(\mathcal{B}_{1}+\mathcal{B}_{2}, \mathcal{B}_{2}-\mathcal{B}_{1}\right)$.

We can extract two groups of fermionic source and the dual operator, which are $\left(B_{+}, A_{+}\right)$and $\left(A_{-}, B_{-}\right)$. The dimensions of the operators are $\frac{3}{2}+m$ and $\frac{3}{2}-m$, respectively [8].

According to the AdS/CFT dictionary, the retarded Green function for the non-relativistic fermionic fixed point can be defined as

$$
\left(\begin{array}{c}
A_{+} \\
B_{-}
\end{array}\right)=G_{R}\left(\begin{array}{c}
B_{+} \\
A_{-}
\end{array}\right)
$$

Following the analysis in [1]], the matric $G_{R}=\left(\begin{array}{cc}\frac{2 G_{1} G_{2}}{G_{1}+G_{2}} & \frac{G_{1}-G_{2}}{G_{1}+G_{2}} \\ \frac{G_{1}-G_{2}}{G_{1}+G_{2}} & \frac{-2}{G_{1}+G_{2}}\end{array}\right)$ is off-diagonal and its eigenvalue $\lambda_{ \pm}$can be expressed in terms of $G_{I}$ as

$$
\lambda_{ \pm}=\frac{G_{1} G_{2}-1 \pm \sqrt{1+G_{1}^{2}+G_{2}^{2}+G_{1}^{2} G_{2}^{2}}}{G_{1}+G_{2}}
$$

Thus, the spectral function has the form

$$
A(\omega, k)=\operatorname{Tr}\left[\operatorname{Im} G_{R}\right]=\frac{2 G_{1} G_{2}-2}{G_{1}+G_{2}} .
$$

\section{NUMERICAL RESULTS}

We numerically integrate the flow equation (11) and read off the asymptotic values to compute the retarded Green functions with the Lorentz violating boundary term in the charged dilatonic AdS black hole background. We will calculate the fermion spectral function and also the density of states. We will investigate the effects of the dipole coupling, the fermion charge and the dilaton field on the holographic fermionic system.

\section{A. Zero temperature}

In this subsection, we will show our numerical results of the massless fermion in the limit of zero temperature.

For convenience, we fix $L=1$. The chemical potential reads $\mu=-\sqrt{3} Q$. 
1. The dipole effect on the flat band and the Fermi surface

We choose $Q=1$ in this subsection. In our computation we first set $q=1$. The numerical results for the spectral function are shown in Figs 14
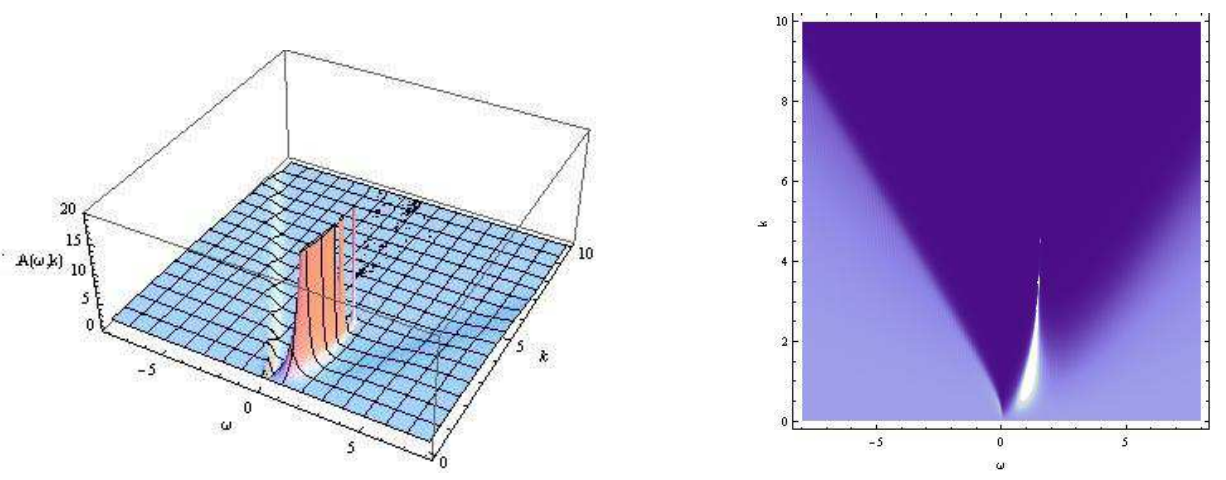

FIG. 1: The plots of $A(\omega, k)$ for the case of $q=1$ and $p=0$.
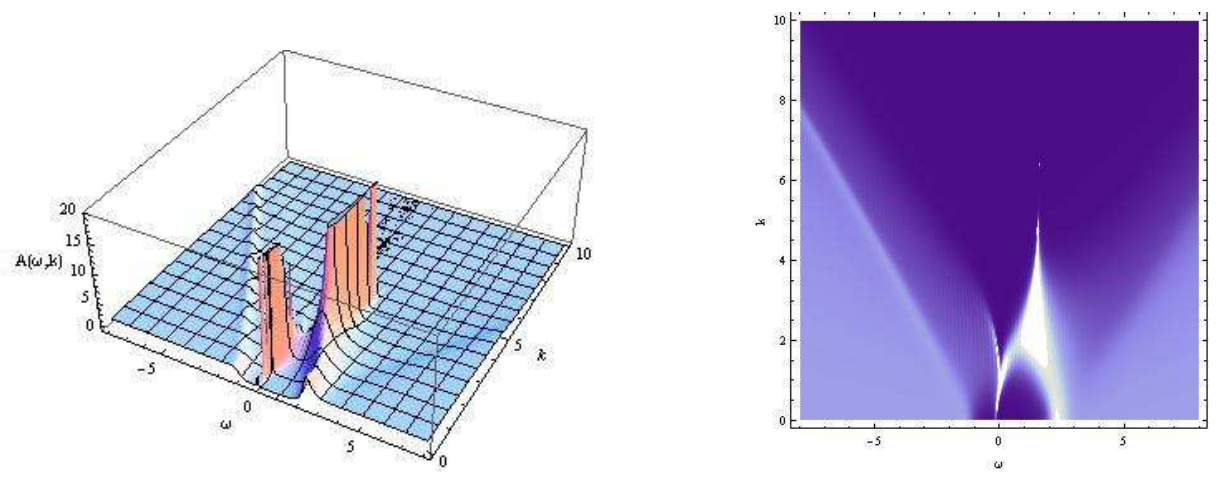

FIG. 2: The plots of $A(\omega, k)$ for the case of $q=1$ and $p=2$.
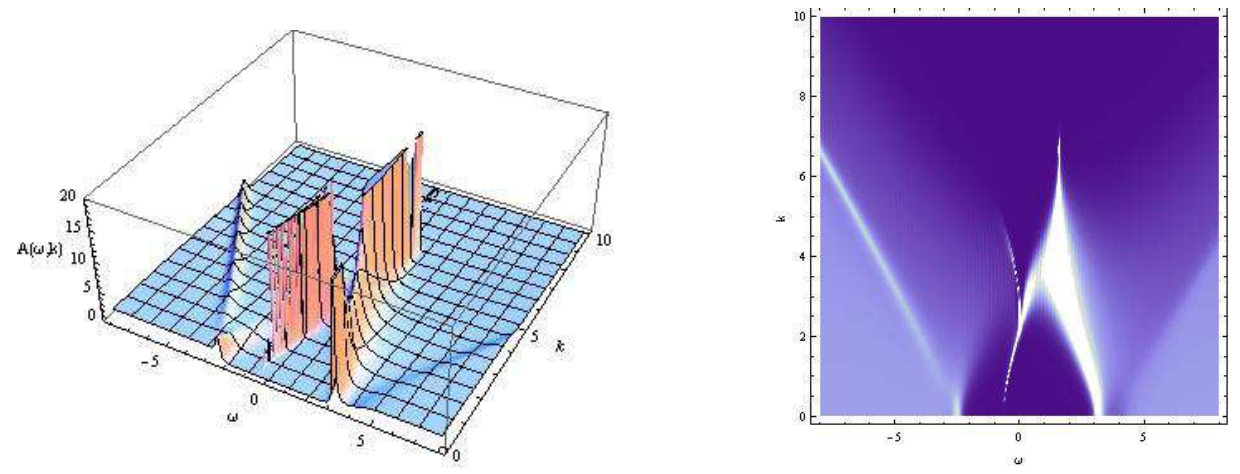

FIG. 3: The plots of $A(\omega, k)$ for the case of $q=1$ and $p=4$.

When there is no dipole coupling between the fermonic field and the gauge field, $p=0$, the result shown in Fig 1 goes back to figure 1 in [11] for the non-relativistic fixed point. Clearly, we see that $A(\omega, k)$ shows the flat band with the peculiar property of poles distributing continuously at a finite interval of momenta. This 

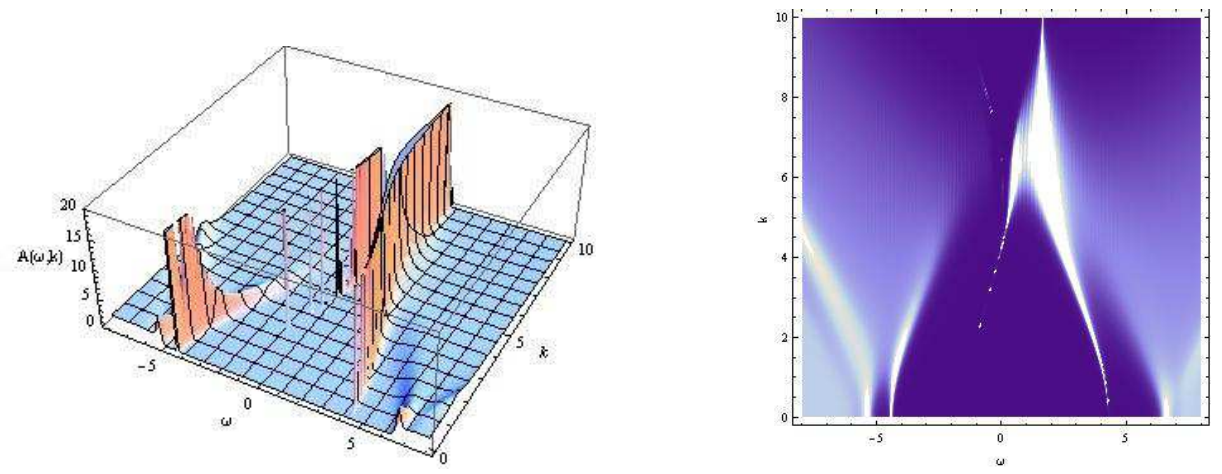

FIG. 4: The plots of $A(\omega, k)$ for the case of $q=1$ and $p=8$.

finite band is mildly dispersed at low momentum, but it presents strong peak at high momentum. This is because the high momentum modes sit outside the lightcone and can't decay. The peak tends sharper as can be checked by plotting a suitable cross section at fixed high $k$.

We now turn on the dipole coupling parameter $p$ and report the results in Figs 2 4t low momentum, the flat band gets more dispersed and the band recovers flatness at higher momentum when the non-minimum fermion coupling becomes stronger. With the increase of the dipole coupling, the frequency of the flat band is larger and the peak of the flat band becomes sharper at small momenta, which means that more energy is needed to excite the small momentum modes for larger $p$. A finite gap opens up and enlarges when the dipole coupling becomes strong. The dipole coupling $p$ drives the dynamical formation of a gap, which mimics the role of interaction strength $U / t$ in the Hubbard model, where the Mott gap forms once $U / t$ exceeds a critical value. The further extreme behavior of the flat band shows the physics approaching a more strongly coupled corner when the dipole coupling becomes stronger. Furthermore we observe that at large enough momentum the flat band always shifts to $\omega=\sqrt{3}$ for all dipole coupling. This is because the frequency $\omega$ is measured relative to the chemical potential. The flat band corresponds to some zero modes in the Minkowski vaccum, with the vanishing absolute energy of Fermion characterized by $\omega_{\text {eff }}=\omega+q A_{t}$ in the Dirac equation (5) on the boundary. From (2) (44), it is clear that $A_{t}=\mu$ at the boundary so that the frequency $\omega$ relates to the chemical potential at the boundary, which is independent of the dipole coupling.

The appearance of the flat band for the non-relativistic fermions observed here is interesting, which has not been observed for the relativistic fermions in the charged dilatonic AdS black hole background [24]. The different properties between the non-relativistic and relativistic fermions observed here support the findings in [10] by comparing with [21] for the charged AdS black hole background.

Now we turn to discuss the fermi momentum. From Fig 14 we see that the Fermi surface opens up for 


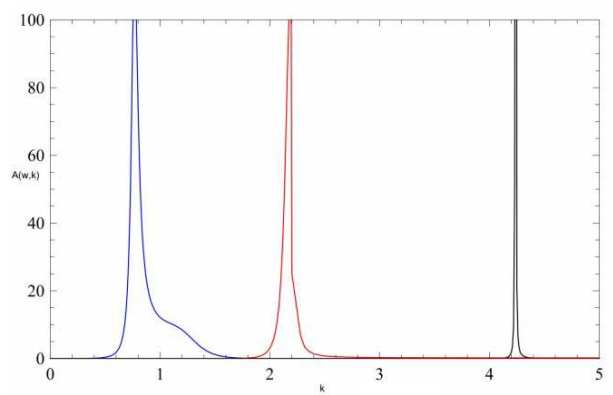

FIG. 5: The function of $A(\omega, k)$ at $\omega=-10^{-7}$ for different dipole coupling. The lines from left to right are for $p=2,4$ and 8 , respectively. In the computation we take $q=1$.

\begin{tabular}{|c|c|c|c|c|}
\hline$p$ & 0 & 2 & 4 & 8 \\
\hline$k_{F}$ & $\mathrm{No}$ & 0.76806283 & 2.19010221 & 4.23765084 \\
\hline$v_{F}$ & $\mathrm{No}$ & 0.286127 & 0.444296 & 0.401080 \\
\hline
\end{tabular}

TABLE I: The Fermi momentum and Fermi velocity for different dipole couplings when we choose $q=1$.

nonzero $p$. In the limit $\omega \rightarrow 0$, the sharp peak of the spectral function represents the fermi surface. For various values of $p$ we illustrate the location of Fermi surface in Fig 5 . We find that the corresponding Fermi momentum increases when the dipole interaction becomes stronger as shown in Table】 We can further investigate the dispersion relation near the Fermi surface. For various $p$ we show in Fig 6 that the linear relation between $\omega$ and $k_{\perp}=k-k_{F}$ behaves as

$$
\omega \simeq v_{F} k_{\perp}
$$

This linear dispersion relation indicates that the excitation near the Fermi surface is well-defined and the Fermi liquid is like the Landau Fermi liquid. This property is not influenced by the dipole coupling, which is consistent with the case of relativistic fermions discussed in [24]. The values of the Fermi velocity $v_{F}=\frac{\partial \omega}{\partial k}$ for different dipole coupling are listed in Table!

As we discussed above that the bulk dipole coupling indeed has imprint on the holographic system. It is natural to ask its role in the properties of the fermion operator in the dual theory. In the vacuum, the coupling does not affect the fermion-fermion Green function. However, it will influence the algebraic structure of the current-fermion-fermion three point correlation function. Though the full story of the dipole effect from CFT is complicated, one simple character we can see from $\mathbb{\mathbb { 1 }}$ is that when $p=0$, there are no terms in $\left\langle\bar{\zeta}_{\alpha} J^{\mu} \zeta_{\beta}\right\rangle$ proportional to the second rank clifford algebra element $\Gamma^{\mu \nu}$, while for nonzero dipole couplings, such terms will arise [30]. As the dipole coupling increases, this makes the Fermi surface pole fall into a log-oscillatory region [16], disappear and finally a gap forms. It would be interesting to analytically understand why the dipole coupling change the shape of the Fermi surface and the oscillatory region and further explore the full 

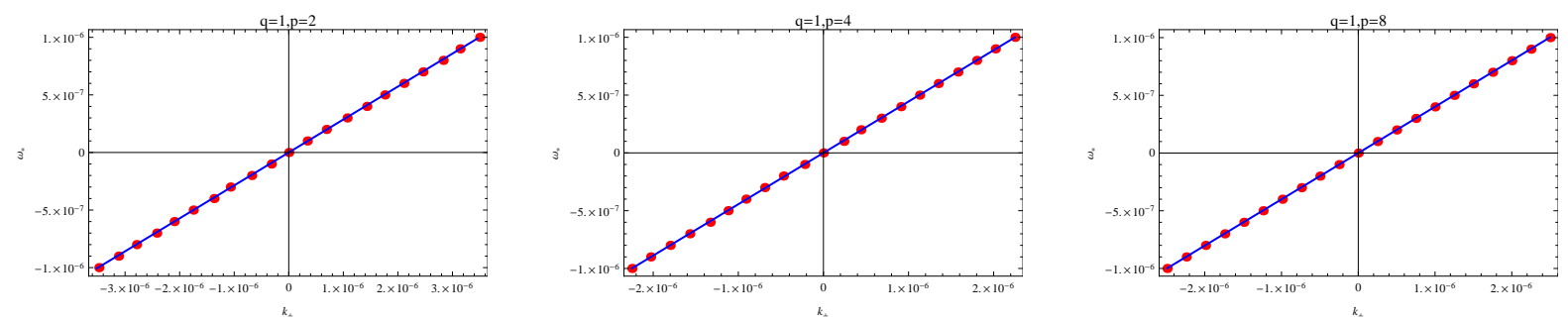

FIG. 6: The dispersion relation near the Fermi surface at the Non-relativistic fixed point of $q=1$ for various $p$.
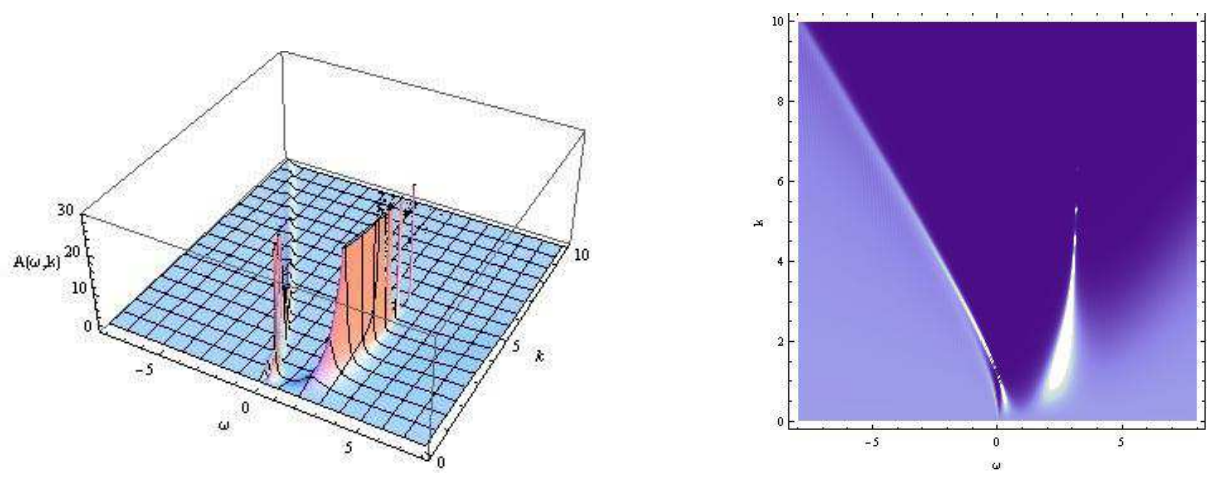

FIG. 7: The plots of $A(\omega, k)$ for the case of $q=2$ and $p=0$.

story in the field theory. This will serve as a separate work in the future.

2. The influence of fermion charge and the dilaton field

The chemical potential felt by the probe fermion is read as $\Omega=q \mu$. Since $g_{F}$, the effective dimensionless gauge coupling, is implied in $\mu$, we have $\Omega \sim g_{F} q$. In the conformal field theory, the fermion charge $q$ or the gauge coupling $g_{F}$ denotes the interaction strength between the spinor and massless vector fields. It will modify the $n$-point function for the boundary spinors by bringing the vector-spinor-spinor function. The detailed calculation from the AdS/CFT correspondence with this interaction was shown in [31]. The authors of 16] have reported that the properties of the holographic fermion are charge dependent. On the other hand, here from the bulk action, we can see that the dilaton field is related to the gauge coupling $g_{F}$ through the form $g_{F}^{2}=4 e^{-\phi}$. This shows that the dilaton field affects the gauge coupling and in turn will have imprint on the chemical potential of the boundary field theory. Thus, it is of interest to study how the fermion charge and dilaton field affect the spectral function, respectively.

In [32], it was claimed that the charge $q$ can affect the excitation near the Fermi surface in charged dilaton black hole. In addition, it was argued that there is some kind of competition between the charge $q$ and the dipole coupling $p$ to create the Fermi surface for the non-relativistic fixed point in charged AdS black hole 11]. Here we would like to further examine the effect of $q$ in the Fermi system in the charged dilatonic AdS 

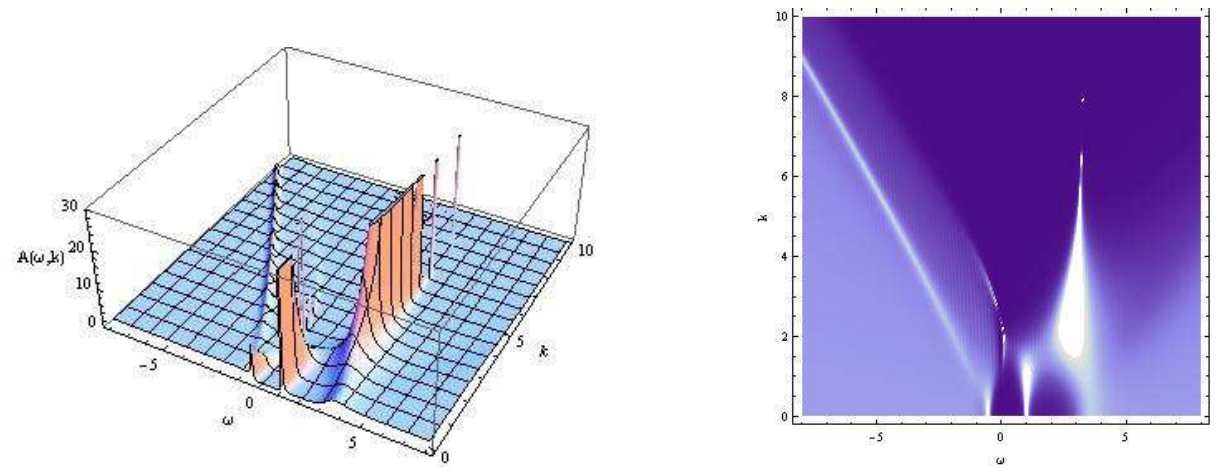

FIG. 8: The plots of $A(\omega, k)$ for the case of $q=2$ and $p=2$.
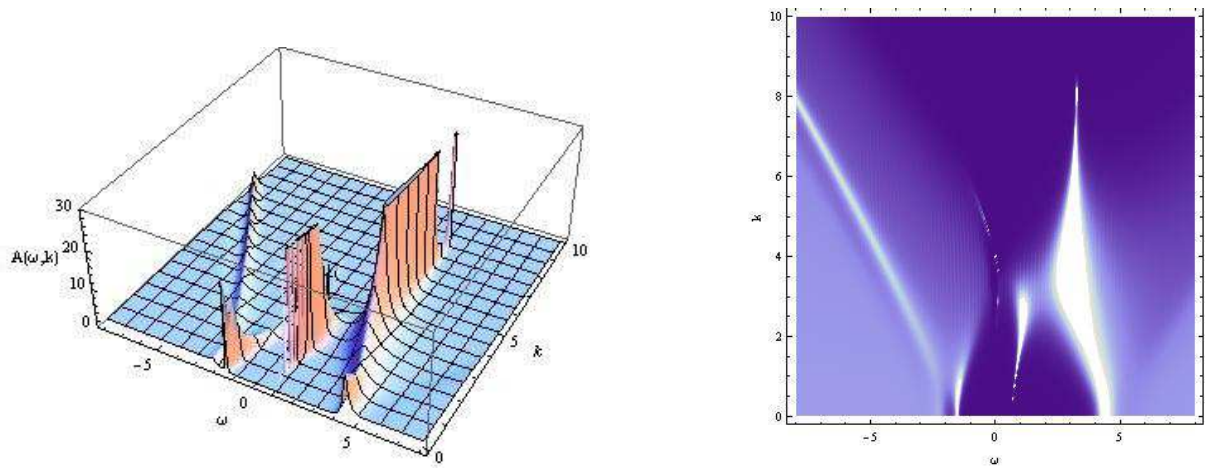

FIG. 9: The plots of $A(\omega, k)$ for the case of $q=2$ and $p=4$.

background. We will change the value of $q$ in the computation and compare with the result for $q=1$. The spectral functions are shown in Fig[7][10 for taking $q=2$. Similar to the results by setting $q=1$, flat bands tend to $2 \sqrt{3}$ at large enough momentum which is independent of the dipole coupling. Besides the similarity, we observe the different property for choosing different $q$. When $q=2$, we see that the quasi-particle like peak, i.e., Fermi surface appears even at $p=0$. This is different from small $q$ case where there is no Fermi surface in the $\omega=0$ limit for the minimal Fermion coupling. Moreover, the emergence of the gap can happen at smaller dipole coupling when $q$ is bigger, for example the gap emerges around $p \sim 13$ for $q=1$, while the gap appears around $p \sim 8.5$ when $q=2$. These observations support that $q$ is nontrivial, it influences the Fermi system.

The Fermi momentum and Fermi velocity for choosing $q=2$ are listed in Table $\Pi$ Similar to setting $q=1$, the Fermi momentum increases with $p$ and the dispersion relation is kept linear. We see that the

\begin{tabular}{|c|c|c|c|c|}
\hline$p$ & 0 & 2 & 4 & 8 \\
\hline$k_{F}$ & 1.18628543 & 2.33984327 & 3.99854464 & 7.40908575 \\
\hline$v_{F}$ & -0.607729 & -0.380546 & -0.380714 & -0.382852 \\
\hline
\end{tabular}

TABLE II: The Fermi momentum and Fermi velocity for different dipole couplings for $q=2$. 

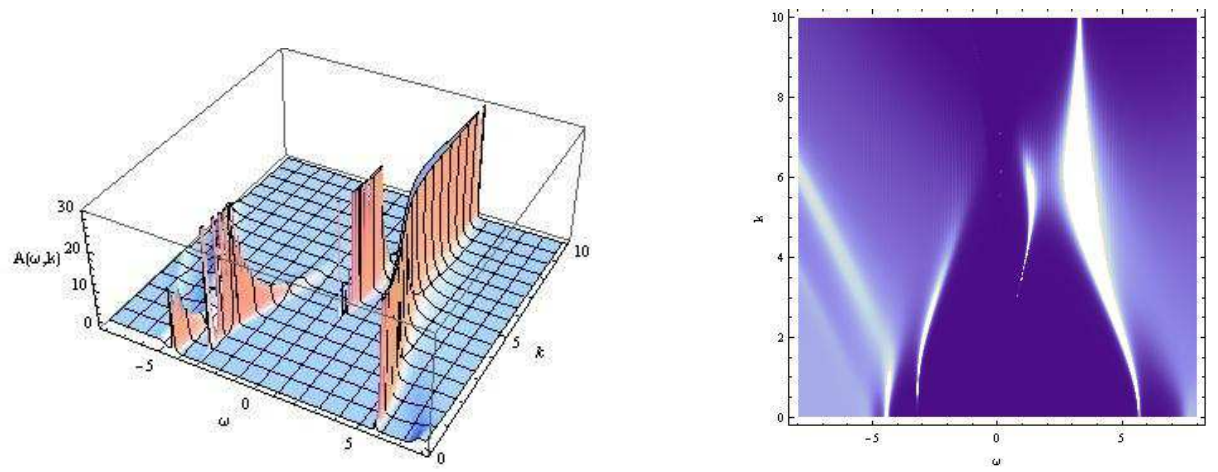

FIG. 10: The plots of $A(\omega, k)$ for the case of $q=2$ and $p=8$.

dispersion relation is always linear and is independent of the fermion charge and the dipole coupling in this case. Comparing with the relativistic situation 24], we find that for the same bulk coupling, the Fermi momentum is smaller in the non-relativistic case. The suppression of the Fermi momentum in the nonrelativistic case can attribute to the presence of the flat band. In addition, we find that the Fermi velocities for $q=2$ and $q=1$ have opposite signs. These imply that the charge $q$ do influence the excitation near the Fermi surface which supports the claim in [32].

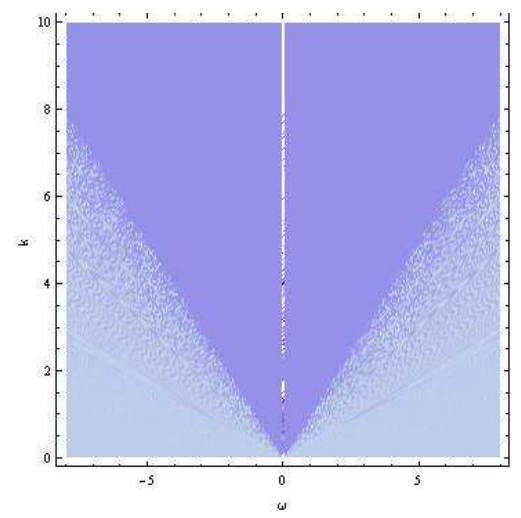

FIG. 11: $A(\omega, k)$ with $q=p=2$ for $Q=0$.
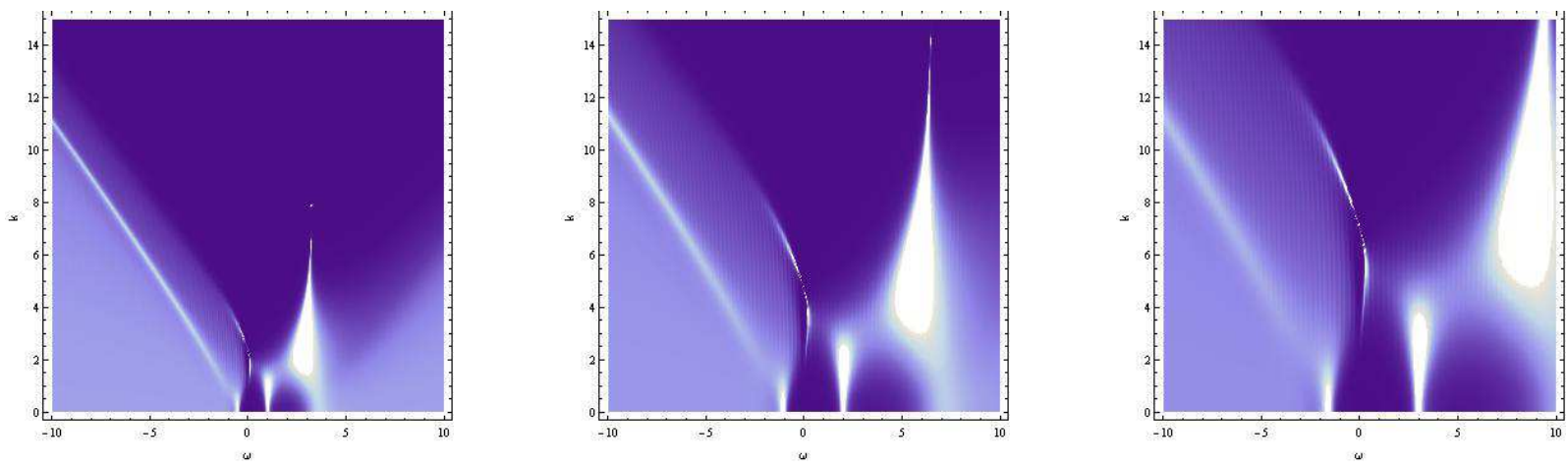

FIG. 12: The plots of $A(\omega, k)$ with $q=p=2$. Here $Q$ is set to be 1,2 and 3 respectively from left to right. 


\begin{tabular}{|c|c|c|c|c|}
\hline$Q$ & 0 & 1 & 2 & 3 \\
\hline$k_{F}$ & $\mathrm{No}$ & 2.33984327 & 4.67974416 & 7.01962931 \\
\hline
\end{tabular}

TABLE III: The Fermi momentum for different $Q$ with $q=p=2$.

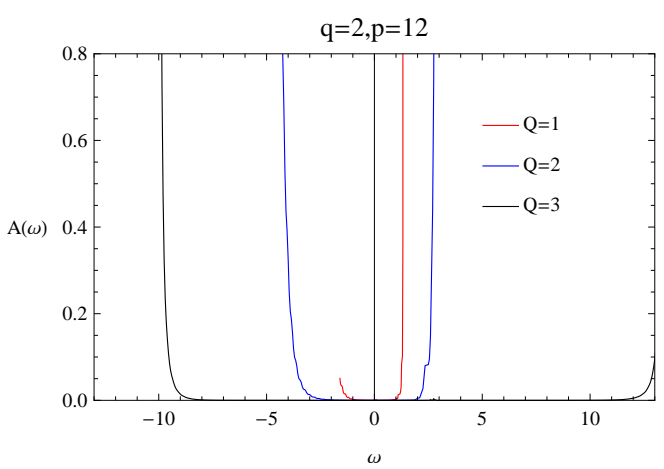

FIG. 13: $A(\omega)$ for different values of $Q$ at zero temperature.

Now we will turn to investigate how the dilaton field influence the behavior of the spectral function. Without loss of generality, we set $q=p=2$ in the discussion. When the dilaton field vanishes $Q=0$, the background (2) goes back to the AdS Schwarzschild black hole. The spectral function for $Q=0$ is shown in Fig 11. This background is not charged, so that it does not have Fermi-like peak.

In the dilaton gravity, the chemical potential is finite as described in (4). This gives the possibility of fermionic excitation. The numerical results on the spectral function for nonzero values of $Q$ are shown in Fig 12. We observe the Fermi-like peak and present the Fermi momentum in Table increases but the peak becomes lower as the increase of the dilaton field $Q$. For larger $Q$, the flat band gets more dispersed in the low momentum and the peak of the band appears at higher momentum. This shows that the dilaton field has the effect on the shift of the flat band.

To see clearly the effect of the dilaton field on the gap, we plot the density of state by integrating $A(\omega, k)$ over $k$ with strong dipole coupling $p=12$ in Fig 13. We observe that with the increase of $Q$, the gap becomes wider. This tells us that in the dilaton gravity the effect of the dipole coupling will be more explicit.

\section{B. Finite temperature}

In 22, 24], it was argued that the gap brought by strong dipole coupling will vanish once the temperature increases to a critical value for the relativistic fermionic system. It is of interest to examine this property for the non-relativistic situation by imposing Lorentz breaking boundary conditions.

We calculate the spectral function at the finite temperature. We set $q=2$ and $p=12$ in the following 

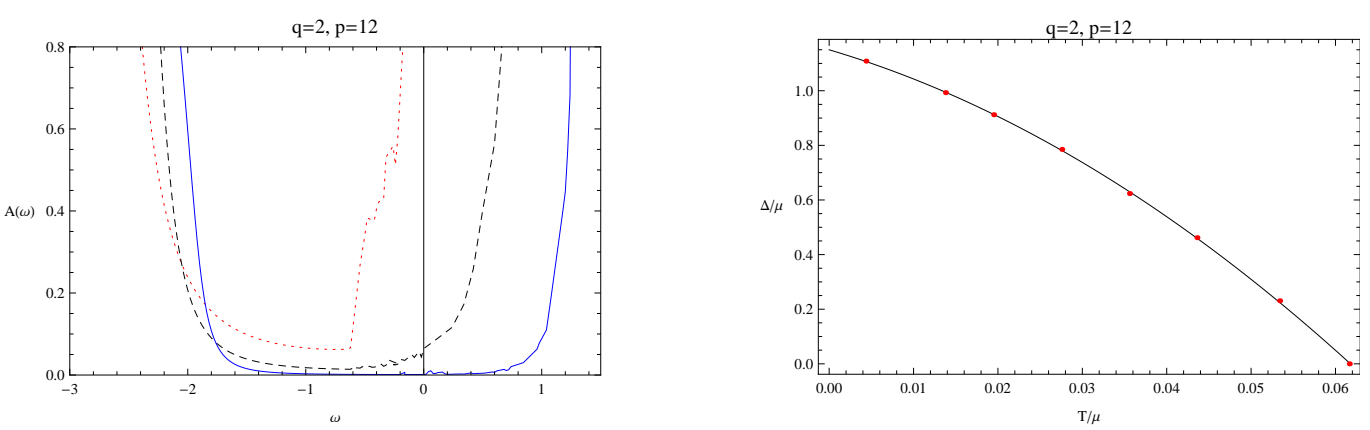

FIG. 14: Left: $A(\omega)$ as a function of $\omega$ when $Q=1$ at finite temperatures. $T / \mu$ are $0.0435864($ solid), $0.0616404($ dashed) and 0.0754938 (dotted). Right: The width of the gap as a function of the temperature.

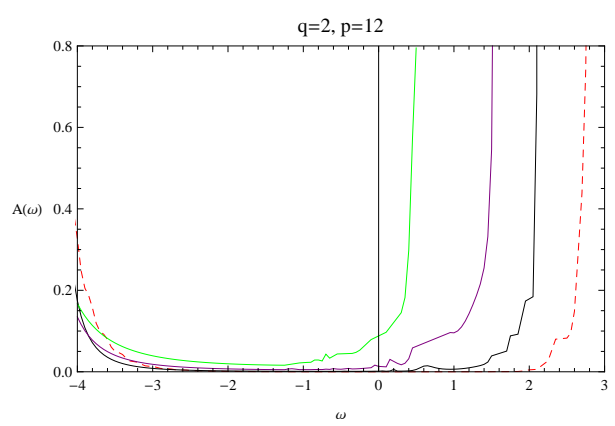

FIG. 15: $A(\omega)$ as a function of $\omega$ when $Q=2$ at finite temperatures. $T / \mu$ are 0(red dashed), 0.0435864(black), 0.0533822 (purple) and 0.0616404 (green), respectively.

discussion. In the left panel of Fig 14 we show the density of state for different temperatures when the dilaton $Q=1$. We see that the gap becomes narrower and closes up when $T / \mu$ increases to the value 0.061 . Moreover, we show the width of the gap $\Delta$ as a function of $T$ in the right panel of Fig [14. As the temperature increases to $T_{\star}$ when the gap closes up, there is a transition from the insulator to conducting state. In our model, We find the ratio $\Delta_{\star} / T_{\star}$ is around 18.65 , where $\Delta_{\star}$ is the width of the gap at zero temperature. This supports that the generated gap is temperature-dependent.

We also calculate the spectral function for different dilaton charge, for example taking $Q=2$. The density of state for different temperature is shown in Fig 15. The property that gap becoming narrower for higher temperature holds as well. For bigger dilaton charge, we find that the value of $\Delta_{\star} / T_{\star}$ is higher, where $T_{\star}$ is the temperature to close up the gap. When $Q=2$, the value of $\Delta_{\star} / T_{\star}$ is around 27.82. This ratio increases with the dilaton charge. Note that in the charged AdS black hole background, the ratio $\Delta_{\star} / T_{\star}$ was found around 10 [22]. It is worth pointing out that similar to the case of zero temperature, the gap becomes wider as we increase $Q$ for fixed finite temperature, this is explicit by comparing Fig 14 and Fig 15 


\section{CONCLUSION AND DISCUSSION}

In this paper, we have studied extensively the properties of the holographic non-relativistic fermionic spectral function in the presence of a bulk dipole coupling in the charged dilatonic black hole background. We generalized previous related studies [11, 24] by combining non-relativistic fermions, dipole coupling and a dilaton field and studied how all these ingredients affect together the properties of this fermionic system.

Firstly, we have further confirmed that the emergence of flat band is robust in the case of non-relativistic fermionic fixed point, independent of the black hole background and the coupling between the fermions and gauge field. Secondly, we observed that the Fermi momentum increases as the dipole coupling becomes stronger for some specific charge $q$ and small $p$. By studying the dispersion relation for small $p$, we found that this fermionic system had the linear dispersion relation for these specific charge $q$ and $p$. Then, by studying the large dipole coupling effects, we found that for a fixed $q$, the Fermi sea disappeares and a gap opens up when the dipole coupling goes beyond a critical value, which indicated this fermionic system possesses the characteristic of the Mott insulator. Our calculations also showed to us that bigger charge make the gap opens easier. Subsequently, we also investigated how the dilaton field influence the behavior of the spectral function. The results showed that the effect of the dipole coupling is more explicit in the dilaton gravity. Finally, we found that this holographic non-relativistic fermionic system with dipole coupling also exhibits a phase transition from insulator to a conducting state as the temperature is increased. It implied that the gap emerged by the dipole coupling in this dilaton background possesses non-trivial temperature dynamics.

The properties for the nonrelativistic fermion system disclosed here in the holographic study with the modification of the boundary term are interesting. It is of interest to further explore the holographic properties of the nonrelativistic fermion system, for example the backreaction of the bulk fermion on the background, the nature of the Fermi surface and its relation to the phase transition etc. Besides, it will be interesting

to examine whether the model satisfies the Luttinger's theorem [33 35]. It is expected that the theoretical attempts in this direction can help to unlock more puzzles in the condense matter physics.

\section{Acknowledgments}

X. M. Kuang and B. Wang are supported partially by the NNSF of China and the Shanghai Science and Technology Commission under the grant 11DZ2260700. J. P. Wu is partly supported by the National Research Foundation of Korea(NRF) grant funded by the Korea government(MEST) through the Center for Quantum 
Spacetime(CQUeST) of Sogang University with grant number 2005-0049409 and also by the NSFC under grant No.11275208.

[1] S.A. Hartnoll, Class. Quant. Grav. 26, 224002 (2009), arXiv:0903.3246].

[2] C.P. Herzog, J. Phys. A 42, 343001 (2009), arXiv:0904.1975.

[3] G.T. Horowitz, [arXiv: 1002.1722].

[4] S. S. Gubser, F. D. Rocha, Phys. Rev. D 81, 046001 (2010), arXiv:0911.2898;

[5] K. Goldstein, S. Kachru, S. Prakash and S. P. Trivedi, JHEP 1008 (2010) 078, arXiv:0911.3586.

[6] C. Charmousis, B. Gouteraux, B. S. Kim, E. Kiritsis and R. Meyer, JHEP 1011 (2010) 151. arXiv:1005.4690.

[7] S. A. Hartnoll, L. Huijse, Class.Quant.Grav. 29 (2012) 194001, arXiv:1111.2606].

[8] J. N. Laia, D. Tong, JHEP 1111 (2011) 125, arXiv:1108.1381.

[9] J. N. Laia, D. Tong, JHEP 1111 (2011) 131 arXiv:1108.2216

[10] W. J. Li, H. Zhang, JHEP1111(2011) 018, arXiv:1110.4559.

[11] W. J. Li, R. Meyer, H. Zhang, JHEP1201 (2012) 153, arXiv:1111.3783.

[12] W. J. Li, J. P. Wu, Nuclear Physics B, 867 (2013), 810-826, arXiv:1203.0674

[13] S. S. Lee, Phys. Rev. D 79, 086006, (2009), arXiv:0809.3402.

[14] N. Iqbal and H. Liu, Phys. Rev. D 79, 025023 (2009), arXiv:0809.3808.

[15] T. Faulkner, H. Liu, J. McGreevy and D. Vegh, Phys. Rev. D 83:125002,2011, [arXir:0907.2694].

[16] H. Liu, J. McGreevy and D. Vegh, Phys. Rev. D 83, 065029 (2011), arXiv:0903.2477.

[17] N. Iqbal and H. Liu, Fortsch. Phys. 57, 367 (2009), arXiv:0903.2596.

[18] M. Cubrovic, J. Zaanen and K. Schalm, Science 325 (2009) 439, arXiv:0904.1993.

[19] J. P. Wu, JHEP 1107:106,2011, arXiv:1103.3982.

[20] L. Q. Fang, X. H. Ge, X. M. Kuang, Phys. Rev. D 86, 105037 (2012), arXiv:1201.3832.

[21] M. Edalati, R. G. Leigh, P. W. Phillips, Phys. Rev. Lett. 106, 091602 (2011), arXiv:1010.3238.

[22] M. Edalati, R. G. Leigh, K. W. Lo, P. W. Phillips, Phys. Rev. D 83, 046012 (2011), arXiv:1012.3751.

[23] J. P. Wu, H. B. Zeng, JHEP 1204 (2012) 068, arXiv:1201.2485.

[24] W. Y. Wen, S. Y. Wu, Phys. Lett. B712 (2012) 266-271, arXiv:1202.6539.

[25] X. M. Kuang, B. Wang, J. P. Wu, JHEP 07 (2012) 125, arXiv:1205.6674.

[26] R. M. Wald, "General Relativity", The University of Chicago Press.

[27] S. S. Gubser and I. Mitra, JHEP 08 (2001) 018, arXiv:hep-th/0011127.

[28] M. Cvetic, M.J. Duff, P. Hoxha et. al., Nucl. Phys. B558 (1999) 96 126, arXiv:hep-th/9903214.

[29] T. Faulkner, G. T. Horowitz, J. McGreevy, M. M. Roberts, D. Vegh, JHEP 1003, 121 (2010), arXiv:0911.3402].

[30] D. Guarrera, J. McGreevy, arXiv:1102.3908.

[31] W. Mück, K. S. Viswanathan, Phys. Rev. D 58:106006,1998, arXiv:hep-th/9805145.

[32] S. S. Gubser, Jie Ren, Phys. Rev. D 86:046004,2012, arXiv:1204.6315].

[33] N. Iqbal and H. Liu, Classical Quantum Gravity 29, 194004 (2012), arXiv:1112.3671.

[34] S. Sachdev, Phys. Rev. D 84, 066009 (2011), arXiv:1107.5321].

[35] A. Allais, J. McGreevy, and S. J. Suh, Phys. Rev. Lett. 108,231602 (2012), arXiv:1202.5308. 